\title{
Absence of a set of plasmid-encoded genes is predictive of reduced pathogenic potential in Brachyspira hyodysenteriae
}

\author{
Tom La', Nyree D Phillips ${ }^{1}$, Jill R Thomson ${ }^{2}$ and David J Hampson ${ }^{1 *}$
}

\begin{abstract}
The gene content of 14 strains of the intestinal spirochaete Brachyspira hyodysenteriae was compared using a DNA microarray. A consistent difference occurred in a block of four genes on the $\sim 36 \mathrm{~Kb}$ plasmid, with these being present in six virulent strains and absent in eight strains with reduced pathogenic potential. These genes encoded a predicted radical S-adenosylmethionine domain protein, a glycosyl transferase group 1-like protein, an NAD dependant epimerase and a dTDP-4-dehydrorhamnose 2-5 epimerase: they may be involved in rhamnose biosynthesis and glycosylation. The absence of these plasmid genes in $B$. hyodysenteriae isolates is predictive of reduced pathogenic potential.
\end{abstract}

\section{Introduction, methods, and results}

The anaerobic intestinal spirochaete Brachyspira hyodysenteriae infects the large intestine of grower and finisher pigs to cause swine dysentery (SD) [1]. In the field signs of SD may be masked or prevented by the use of antimicrobial agents, whilst dietary ingredients may influence the occurrence and severity of disease by creating conditions within the large intestine that inhibit or promote colonisation [2-4]. Differences in the B. hyodysenteriae strains also may account for variations in clinical outcomes. Lysons et al. [5] reported isolating $B$. hyodysenteriae from pigs in herds that were free of clinical SD, and three isolates did not produce disease in experimentally infected pigs, although they colonised the colonic mucosa of some animals. Jensen and Stanton [6] reported that B. hyodysenteriae reference strain B204 colonised more experimentally challenged pigs and was more virulent than the type strain $\mathrm{B}^{\mathrm{T}}{ }^{\mathrm{T}}$. Achacha et al. [7] challenged eight groups of weaner pigs with seven reference strains and one field isolate of $B$. hyodysenteriae, and categorised these as being of high or low virulence. Strain B204 was considered to be virulent, whilst strains B234 and A1 were considered to have low pathogenic potential. Thomson et al. [8] challenged weaner pigs with four $B$. hyodysenteriae isolates from herds with only mild diarrhoea or low prevalence of

\footnotetext{
* Correspondence: d.hampson@murdoch.edu.au

${ }^{1}$ School of Veterinary and Life Sciences, Murdoch University, Murdoch, Western Australia 6150, Australia

Full list of author information is available at the end of the article
}

disease. Fewer of the pigs challenged with these strains showed clinical signs, pathological changes or reduced growth rates compared to pigs challenged with isolates from herds with more severe disease. Host species of origin also may be involved in differences in pathogenesis, as B. hyodysenteriae strains R1 and R358 isolated from common rheas (Rhea americana) with severe typhlitis failed to colonise pigs [9].

There have been few attempts to determine why isolates of $B$. hyodysenteriae vary in their pathogenic potential. Analysis of the complete genome sequence of virulent $B$. hyodysenteriae strain WA1, including its $\sim 36 \mathrm{~Kb}$ plasmid, identified $314(\sim 12 \%)$ predicted open reading frames (ORFs) as having potential roles in the pathogenesis of SD [10]. These included "life style" genes such as those associated with motility and chemotaxis, those encoding surface proteins and enzymes involved in the synthesis of lipooligosaccharide (LOS), and a number of genes predicted to encode proteolytic enzymes and haemolysins/cytotoxins. Subsequent evidence for a role of the $36 \mathrm{~Kb}$ plasmid in pathogenesis was obtained when fewer pigs developed SD after experimental challenge with a $B$. hyodysenteriae strain lacking the plasmid compared to pigs challenged with a strain containing the plasmid [11]. The aim of the current study was to identify genes involved in pathogenesis by comparing the gene content of strains of B. hyodysenteriae with high or low pathogenic potential using a microarray analysis. 
Spirochaete strains were obtained as frozen stock from the culture collections at Murdoch University and the Scottish Agricultural College. The B. hyodysenteriae strains used in the microarray analysis were selected to include six that have been reported in the literature to be virulent (B204, BW1, NSW5, NSW15, Q17 and Vic2) and eight that have been reported to colonise fewer pigs and/or cause milder disease (A1, B78 ${ }^{\mathrm{T}}, \mathrm{B} 234, \mathrm{~B} 6933, \mathrm{FM} 88.90$, R301, SA2206 and VS1) [5-7,9]. These are recorded as "avirulent", although they are not fully avirulent. Four other strains (P252/97; P257/97; P935/1/00; P44/15/00) that were considered to have low pathogenic potential based on field observations and results of experimental infection of pigs [8] subsequently were used in a PCR analysis of the plasmid genes to help confirm the findings from the microarray work.

Spirochaetes were propagated in Kunkle's pre-reduced anaerobic broth [12], and at a density of approximately $10^{8}$ cells $/ \mathrm{mL}$ they were harvested by centrifuging at $10000 \times g$, and counted under a phase contrast microscope.

Custom GeneChips were designed and manufactured by Affymetrix (Santa Clara, California) using the predicted ORFs from B. hyodysenteriae WA1 [10]. A total of 1993 of the 2638 ORFs encoded on the genome and 24 of the 31 ORFs encoded on the plasmid were represented on the chip. Each ORF was represented on the array by a series of 13 different pairs of 25-mer oligonucleotide probes. Each probe pair consisted of a perfect match oligonucleotide and a mismatch oligonucleotide. The probe set was pruned against all other B. hyodysenteriae WA1 sequences on the chip and the Affymetrix Standard Pruning Libraries for Escherichia coli and Pseudomonas spp. genomes.

Microarray-based comparative genomic hybridisation (CGH) analysis was used to compare the gene content of the six virulent and eight "avirulent" $B$. hyodysenteriae strains with the gene content of virulent $B$. hyodysenteriae strain WA1. A single channel microarray analysis was performed using the Affymetrix GeneChips. High molecular weight DNA was extracted from the B. hyodysenteriae cells using the DNeasy Blood and Tissue Kit (Qiagen) according to the manufacturer's instructions. The integrity of all DNA preparations was confirmed by PCR using species-specific primers [13]. Purified DNA was digested with $R s a 1$ and the restriction fragments were labelled with a fluorescent cyanide dye (Cy3) using the BioPrime Array CGH Genomic Labelling System (Invitrogen). The labelled genome fragments were hybridised to the B. hyodysenteriae GeneChip under moderately stringent conditions $\left(37^{\circ} \mathrm{C}\right)$ in a Hybridisation Oven 645 (Affymetrix) for $16 \mathrm{~h}$. The GeneChips were washed and labelled using the GeneChip Hybridisation, Wash and Stain Kit (Affymetrix), using a Fluidics Station 450 (Affymetrix). The GeneChips were scanned using the Scanner 3000 (Affymetrix) and the hybridisation intensity data converted to numeric values using the GeneChip Operating Software (GCOS, Affymetrix). Affymetrix default software cut-off values were used and each ORF was categorized as "Present", "Marginal" or "Absent". B. hyodysenteriae strain WA1 and B. innocens strain 4/71 served as positive and negative controls, respectively.

For the seven plasmid-encoded genes absent from the GeneChip, three primer pairs were designed for PCR amplification of each (Table 1). For the four genes present in the plasmid of the virulent strains and absent in the "avirulent" strains (BHWA1_02678 to BHWA1_02681), three unique primer pairs were designed for the PCR amplification of each ORF (Table 2). High molecular weight DNA from all strains was subjected to PCR using HotStarTaq DNA Polymerase (Qiagen) according to the manufacturer's instructions. The annealing temperature used for each primer was set at $5{ }^{\circ} \mathrm{C}$ less than the optimal annealing temperature to allow for a moderate stringency similar to that of the microarray hybridisation. The amplification products were electrophoresed through an agarose gel, stained with ethidium bromide and viewed over ultraviolet light.

For the four strains that were not hybridised on the microarray, spirochaetes were harvested from $1 \mathrm{~mL}$ of culture by centrifugation and the pellet was resuspended $1 \mathrm{~mL}$ sterile TE buffer. The resuspended cells were boiled for $2 \mathrm{~min}$, the cellular debris pelleted by centrifugation and the supernatant subjected to PCR.

The categorised values for the $\mathrm{CGH}$ analysis and the PCR results for the genes missing from the microarray were merged. Genes which were present or marginal in the virulent strains but absent in the low-virulence strains were identified. None of the plasmid genes gave marginal signals.

In CGH analysis B. innocens $4 / 71$ shared only 328 of the 1993 WA1 genes, whilst the six virulent $B$. hyodysenteriae strains shared between 1919 and 1982 (96.3 to 99.4\%) of the genes and 1871 (93.9\%) genes were common to all virulent strains (ie were core genes). The eight "avirulent" strains shared 1874 to 1930 (90.4 to $96.8 \%)$ of the WA1 genes, of which 1757 (88.2\%) were common to all the "avirulent" strains. All 14 strains shared 1741 (87.4\%) of the WA1 genes. Only five genes (of unknown function) were specific to B. hyodysenteriae WA1 (BHWA1_01317, BHWA1_00005, BHWA1_00006, BHWA1_01358 and BHWA1_00574). The data from the CGH analysis have been deposited at the Gene Expression Omnibus public repository [14].

The CGH analysis identified four genes that were present in all six virulent strains (and WA1) and absent in all eight "avirulent" strains. These were located as a block of adjacent genes on the $36 \mathrm{~Kb}$ plasmid (positions 13-16), and were predicted to encode a radical S-adenosylmethionine (SAM) protein, a glycosyltranserase, an NAD-dependent 
Table 1 PCR primers used for the amplification of B. hyodysenteriae plasmid genes not on the GeneChip

\begin{tabular}{|c|c|c|c|c|c|}
\hline Primer name $^{\mathrm{a}}$ & Sequence $\left(5^{\prime}-3^{\prime}\right)$ & Product size & $\begin{array}{l}\text { B. hyodysenteri } \\
\text { strains }\end{array}$ & strains but present in all & virulent \\
\hline BHWA1_02666a-F & gagaagaatatttaatacctttaatag & $217 \mathrm{bp}$ & Primer name ${ }^{b}$ & Sequence $\left(5^{\prime}-3^{\prime}\right)$ & Product size \\
\hline BHWA1_02666a-R & callicaldaldadaldaldatgyilyg & $549 \mathrm{hn}$ & BHWA1_02678a-F & tagaaacggtaatcccattag & 322 bp \\
\hline BHWAI_02666b-F & & $349 \mathrm{NP}$ & BHWA1_02678a-R & taaaagcgaagcattagtagtag & \\
\hline DTVVAI_ULOOOD-K & 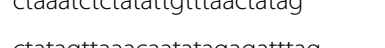 & $177 \mathrm{hno}$ & BHWA1_02678b-F & tgataaagtaaatagggtagatactactac & $420 \mathrm{bp}$ \\
\hline 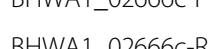 & 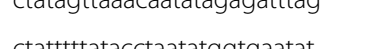 & (17) & BHWA1_02678b-R & aaatacataaggacaaaccattac & \\
\hline 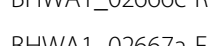 & 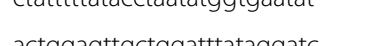 & $560 \mathrm{bn}$ & BHWA1_02678c-F & ttttgctgaaatggtagagtatg & $558 \mathrm{bp}$ \\
\hline 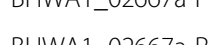 & 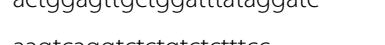 & 200 & BHWA1_02678c-R & tatagtttttactgattctttattgacatc & \\
\hline 年 & 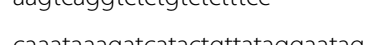 & $507 \mathrm{hn}$ & BHWA1_02679a-F & ttggtggaggagttggtac & $237 \mathrm{bp}$ \\
\hline BHWA1 $02667 \mathrm{~b}-\mathrm{B}$ & 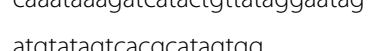 & 的 & BHWA1_02679a-R & tgataaagaagaggatgattcc & \\
\hline BHWA1 $02667 c-F$ & alglatagledegedtaglyg & $204 \mathrm{hot}$ & BHWA1_02679b-F & tcaggtataaatccgcctaatg & $495 \mathrm{bp}$ \\
\hline BHWAI_OZOO/C-F & tgtaatacatttagcaggatatgg & $384 \mathrm{DP}$ & BHWA1_02679b-R & caggtactaaaccagcagtcatag & \\
\hline 年 & 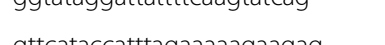 & & BHWA1_02679c-F & ggttttatatgtaagaatgaagatg & $329 \mathrm{bp}$ \\
\hline BHWA1 02668 -B & gllcaldccallidydddadyadyay & & BHWA1_02679c-R & cttagaatttgaagtccagtttg & \\
\hline 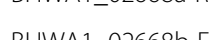 & אนา & $06 \mathrm{~b}$ & BHWA1_02680a-F & gaagttttggcataggaac & $289 \mathrm{bp}$ \\
\hline 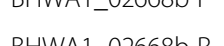 & 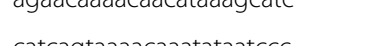 & $200 \mathrm{Nop}$ & BHWA1_02680a-R & tcattttcttttaatggtgtatc & \\
\hline BHWA 102668 C-F & 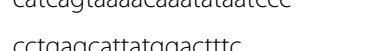 & $240 \mathrm{hn}$ & BHWA1_02680b-F & aaaaggctatttagaatctgaag & $280 \mathrm{bp}$ \\
\hline $02668 c-R$ & tatactatctactettetatagt & & BHWA1_02680b-R & cattatctccataagtatagaaaactctac & \\
\hline DIIVAAI_OZOUOC-n & a & $117 \mathrm{hn}$ & BHWA1_02680c-F & tggtgatatagcaaaaagtatagc & $183 \mathrm{bp}$ \\
\hline BHWVAI_O $20 / \angle \mathrm{La}-\mathrm{T}$ & daatgragaagatattgrattgec & 41/ DP & BHWA1_02680C-R & ccctacaataattttaggttcttcag & \\
\hline 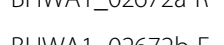 & acticticlatatgittulatact & $16 \mathrm{hn}$ & BHWA1_02681a-F & gtgggaaaaataaatctgaag & $345 \mathrm{bp}$ \\
\hline RHU:A $102672 \mathrm{~b}$ R & attactacaaaatgtactctaaaatgtaag & $540 \mathrm{dP}$ & BHWA1_02681a-R & gcttaattctacagtaaaatgctg & \\
\hline & dClalatgacaddaddtaddalclag & $108 \mathrm{~b}$ & BHWA1_02681b-F & gtaatgatgaacttaaaaaatcaggtattg & $266 \mathrm{bp}$ \\
\hline BHWAI_026/2C-F & tatctaagtataaaaaacatataggagagg & $498 \mathrm{DP}$ & BHWA1_02681b-R & attccatgagccacataaggag & \\
\hline BHWA] 02673a-F & aaatacttatcaataatcttagtag & $1819 \mathrm{hn}$ & BHWA1_02681c-F & aaacagtcaaatatgagttatagtgc & $391 \mathrm{bp}$ \\
\hline BHWA1 $02673 a-R$ & tttcatcataagcaaaaataatatc & & BHWA1_02681C-R & aaatctggtatacttttatccttttc & \\
\hline
\end{tabular}

\begin{tabular}{ll} 
BHWA1_02673b-R & agattgtcttgacgaataaaag \\
BHWA1_02673c-F & aataaatatgacattaaaggaataaaaatc \\
\hline
\end{tabular}

BHWA1_02673c-R ctattgttagtagcaaaataataaaaatac

Table 2 PCR primers for amplification of the four genes on the plasmid that were absent in the "avirulent" $B$. hyodysenteriae strains but present in all the virulent strains

${ }^{\mathrm{b}}$ Primers named according to the plasmid gene they are designed to amplify.

epimerase and an dTDP-4-dehydrorhamnose 3,5-epimerase $(r f b C)$, which are all catalytic enzymes potentially involved in LOS biosynthesis and/or glycosylation (Table 3). Two adjacent plasmid genes (positions 11 and 12) predicted to encode radical SAM domain proteins also were absent from the eight "avirulent" strains and were present in five of the six virulent strains tested (absent in NSW15). "Avirulent" strains A1 and FM88.90 did not possess the plasmid genes.

Other probe sets identified differences between individual virulent and "avirulent" strains, but these were not consistent. Seven sets identified individual chromosomal genes (of unknown function) in the virulent strains that were absent in six or seven of the eight "avirulent" strains (Table 4). Virulent strain NSW15 lacked four of the genes that were absent in the "avirulent" strains. There were no genes present in all the "avirulent" strains and absent in the virulent strains. 
Table 3 Distribution of the B. hyodysenteriae plasmid genes amongst the virulent and "avirulent" strains determined by GeneChip microarray and PCR

\begin{tabular}{|c|c|c|c|c|c|c|c|c|c|c|c|c|c|c|c|}
\hline \multirow{2}{*}{$\begin{array}{l}\text { Gene (position on } \\
\text { plasmid) }\end{array}$} & \multirow[t]{2}{*}{ Putative function } & \multicolumn{6}{|c|}{ Virulent strains } & \multicolumn{8}{|c|}{ Avirulent strains } \\
\hline & & B204 & BW1 & NSW5 & NSW15 & Q17 & Vic 2 & A1 & B78 & B234 & B6933 & FM88.90 & R301 & SA2206 & VS1 \\
\hline BHWA1_02666 (1) & glycosyltransferase & $P$ & $P$ & $P$ & $P$ & $P$ & $P$ & $A$ & $P$ & $P$ & $P$ & A & $P$ & $P$ & $P$ \\
\hline BHWA1_02667 (2) & NAD dependent epimerase/dehydratase & $P$ & $P$ & $P$ & P & $P$ & $P$ & A & P & P & P & A & $P$ & P & $P$ \\
\hline BHWA1_02668 (3) & glycosyl transferase, family 2 & $P$ & $P$ & $P$ & P & P & $P$ & A & P & P & $P$ & A & $P$ & P & $P$ \\
\hline BHWA1_02669 (4) & glycosyl transferase, group 1-like protein & $P$ & P & P & $P$ & $P$ & $P$ & A & $P$ & $P$ & $P$ & A & $P$ & $P$ & $P$ \\
\hline BHWA1_02670 (5) & glycosyl transferase, group 1 & $P$ & P & $P$ & $P$ & $P$ & $P$ & A & $P$ & $P$ & P & A & $P$ & $P$ & P \\
\hline BHWA1_02671 (6) & glycosyl transferase & $P$ & $P$ & $P$ & $P$ & P & $P$ & A & $P$ & P & P & A & $P$ & P & $P$ \\
\hline BHWA1_02672 (7) & hydrolase (HAD superfamily) protein & $P$ & $P$ & $P$ & $P$ & $P$ & $P$ & $P$ & $P$ & $P$ & $P$ & A & $P$ & $P$ & $P$ \\
\hline BHWA1_02673 (8) & hydrolase (HAD superfamily) protein & $P$ & $P$ & $P$ & $P$ & $P$ & $P$ & $P$ & $P$ & $P$ & $P$ & A & $P$ & $P$ & $P$ \\
\hline BHWA1_02674 (9) & Radical SAM domain protein & $P$ & $P$ & P & P & P & $P$ & $P$ & $P$ & P & $P$ & A & $P$ & P & P \\
\hline BHWA1_02675 (10) & Radical SAM domain protein & $P$ & $P$ & $P$ & $P$ & $P$ & $P$ & $P$ & $P$ & $P$ & $P$ & A & $P$ & $P$ & $P$ \\
\hline BHWA1_02676 (11) & Radical SAM domain protein & P & $P$ & $P$ & A & P & P & A & A & A & A & A & A & A & A \\
\hline BHWA1_02677 (12) & Radical SAM domain protein & $P$ & $P$ & $P$ & A & $P$ & $P$ & A & A & A & A & A & A & A & A \\
\hline BHWA1_02678 (13) & Radical SAM domain protein & P & $P$ & $P$ & $P$ & $P$ & P & $A$ & A & $A$ & A & $A$ & $A$ & $A$ & $A$ \\
\hline BHWA1_02679 (14) & glycosyl transferase, group 1-like protein & $P$ & $P$ & $P$ & $P$ & $P$ & $P$ & A & $A$ & $A$ & A & $A$ & $A$ & $A$ & $A$ \\
\hline BHWA1_02680 (15) & NAD dependent epimerase & $P$ & $P$ & P & $P$ & P & $P$ & A & A & A & A & $A$ & $A$ & A & $A$ \\
\hline BHWA1_02681 (16) & dTDP-4-dehydrorhamnose 3,5-epimerase (rfbC) & $P$ & $P$ & $P$ & $P$ & $P$ & $P$ & A & $A$ & A & $A$ & $A$ & $A$ & A & $A$ \\
\hline BHWA1_02682 (17) & Radical SAM domain protein & $P$ & P & $P$ & $P$ & P & $P$ & A & P & $P$ & P & A & $P$ & $P$ & $P$ \\
\hline BHWA1_02683 (18) & Glucose-1-phosphate cytidylyltransferase (rfbF) & $P$ & $P$ & $P$ & $P$ & $P$ & $P$ & A & $P$ & $P$ & P & A & P & P & P \\
\hline BHWA1_02684 (19) & plasmid partitioning protein (cdsM) & $P$ & $P$ & $P$ & P & P & $P$ & A & $P$ & $P$ & P & A & $P$ & P & P \\
\hline BHWA1_02685 (20) & hypothetical protein & P & P & P & P & P & P & A & $P$ & P & $P$ & A & $P$ & $P$ & $P$ \\
\hline BHWA1_02686 (21) & replicative DNA helicase & $P$ & $P$ & $P$ & P & $P$ & P & A & $P$ & $P$ & $P$ & A & $P$ & $P$ & $P$ \\
\hline BHWA1_02687 (22) & DNA primase-like protein & $P$ & $P$ & $P$ & $P$ & $P$ & $P$ & A & $P$ & $P$ & $P$ & A & $P$ & $P$ & $P$ \\
\hline BHWA1_02688 (23) & integrase & $P$ & $P$ & P & P & P & $P$ & A & $P$ & $P$ & $P$ & A & $P$ & $P$ & $P$ \\
\hline BHWA1_02689 (24) & alpha-1,2-fucosyltransferase & $P$ & A & A & P & A & $P$ & A & $P$ & P & $P$ & A & A & $P$ & $P$ \\
\hline BHWA1_02690 (25) & lipopolysaccharide biosynthesis protein-like protein & $P$ & $P$ & P & P & P & P & A & P & P & P & A & P & P & $P$ \\
\hline BHWA1_02691 (26) & dTDP-D-glucose 4,6-dehydratase (rfbB) & $P$ & P & P & P & P & P & A & P & P & P & A & $P$ & P & $P$ \\
\hline BHWA1_02692 (27) & glucose-1-phosphate thymidylyltransferase (rfbA) & $P$ & P & $P$ & $P$ & $P$ & $P$ & A & P & P & P & A & P & $P$ & $P$ \\
\hline BHWA1_02693 (28) & dTDP-4-keto-L-rhamnose reductase (rfbD) & $P$ & P & P & $P$ & P & $P$ & $A$ & $P$ & P & P & A & $P$ & P & P \\
\hline
\end{tabular}


Table 3 Distribution of the B. hyodysenteriae plasmid genes amongst the virulent and "avirulent" strains determined by GeneChip microarray and PCR (Continued)

\begin{tabular}{|c|c|c|c|c|c|c|c|c|c|c|c|c|c|c|c|}
\hline BHWA1_02694 (29) & dTDP-4-dehydrorhamnose 3,5-epimerase ( $r f b C)$ & $P$ & $P$ & $P$ & $P$ & $P$ & $P$ & A & $P$ & $P$ & $P$ & A & $P$ & $P$ & $P$ \\
\hline BHWA1_02695 (30) & hypothetical protein & $P$ & $P$ & $P$ & $P$ & $P$ & $P$ & A & $P$ & $P$ & $P$ & A & $P$ & $P$ & $\mathrm{P}$ \\
\hline BHWA1_02696 (31) & glycosyltransferase & $\mathrm{P}$ & $\mathrm{P}$ & $\mathrm{P}$ & $P$ & $P$ & $\mathrm{P}$ & A & $P$ & $P$ & $\mathrm{P}$ & A & $P$ & $\mathrm{P}$ & $\mathrm{P}$ \\
\hline
\end{tabular}

The names of seven of the plasmid genes that were not on the microarray (genes 1-3; 7-10) but were amplified by $P C R$ are marked in bold. The microarray results for the four genes (genes 13-16) that were absent in the "avirulent" strains but present in the virulent strains are italicised. Genes 11 and 12 also were absent in the "avirulent" strains and were present in five of the six virulent strains (NSW15 being the exception). in the "avirulent" strains but present in the virulent strains are italicised. Genes 11 and 12 also were absent
$\mathrm{P}$ represents gene present, A represents gene absent. Strains A1 and FM88-90 lacked all 31 plasmid genes. 
Table 4 Other chromosomal genes absent in six or seven of the eight "avirulent" strains but present in five or six of the virulent strains

\begin{tabular}{|c|c|c|c|c|c|c|c|c|c|c|c|c|c|c|c|}
\hline \multirow[b]{2}{*}{ Probe set } & \multirow[b]{2}{*}{ Size (base pairs) } & \multicolumn{6}{|c|}{ Virulent strains } & \multicolumn{8}{|c|}{ Avirulent strains } \\
\hline & & B204 & BW1 & NSW5 & NSW15 & Q17 & Vic2 & B6933 & $\mathrm{B}^{\mathrm{T}} \mathrm{T}^{\mathrm{T}}$ & FM88-90 & $\mathrm{A} 1$ & B234 & VS1 & SA2206 & R301 \\
\hline GeneBh581_at & 318 & $P$ & $P$ & $P$ & $P$ & $P$ & $P$ & A & A & A & $P$ & A & A & A & A \\
\hline GeneBh398_at & 135 & $P$ & $P$ & $P$ & $P$ & $P$ & P & A & A & $P$ & P & A & A & A & A \\
\hline GeneBh1267_at & 117 & $P$ & $P$ & $P$ & $P$ & $P$ & $P$ & A & A & $P$ & A & A & A & A & $P$ \\
\hline GeneBh1690_at & 537 & $P$ & $P$ & $P$ & A & $P$ & P & A & A & A & $P$ & A & A & A & A \\
\hline GeneBh310_at & 93 & $P$ & $P$ & $P$ & A & $P$ & $P$ & M & $P$ & A & A & A & A & A & A \\
\hline GeneBh551_at & 819 & P & $P$ & $P$ & A & $P$ & P & $P$ & A & A & A & A & A & P & A \\
\hline GeneBh513_at & 1176 & $P$ & $P$ & $P$ & A & $P$ & $P$ & $A$ & $A$ & $A$ & $A$ & $A$ & $A$ & $P$ & $P$ \\
\hline
\end{tabular}

All genes are of unknown function. $A$, absent; $P$, present; $M$, marginal (weak signal).

PCR analysis of the seven genes on the plasmid that were not represented on the GeneChip indicated that all the B. hyodysenteriae strains except A1 and FM88.90 possessed all seven genes (Table 3).

When used on the four additional strains of low pathogenic potential that were not tested on the microarray [8], the PCRs targeting plasmid-borne genes $11-16$ failed to amplify them from strain P944/15/10, and failed to amplify the radical SAM genes (genes 10, 11 and 12) from strain P935/1/00.

\section{Discussion}

The "avirulent" strains used in this study were not all completely avirulent, but had been shown to colonise fewer experimental animals after challenge and/or cause milder disease compared to typical field strains. They could be defined as having reduced pathogenic potential compared to typical pathogenic strains.

A striking difference in gene profiles was observed in a block of four genes located on the plasmid which were present in the virulent strains but not in these "avirulent" strains. PCR analysis showed that two adjacent genes encoding radical SAM domain proteins also were absent in the "avirulent" strains, but they were absent in one of the virulent strains as well so that the correlation was less strong.

PCR screening of four additional strains broadly supported the existence of a correlation between the plasmid genes and virulence, although two of the "avirulent" strains possessed these genes and one only lacked the radical SAM domain genes. Clearly reduced virulence may be associated with factors other than absence of the plasmid genes. The fact that the "avirulent" strains used in the study were only able to colonise and induce disease in a small proportion of pigs in previous studies suggests that the genes are most likely to be involved in facilitating aspects of colonisation rather than in lesion development. This could involve specific changes to surface molecules including such things as modification to LOS composition or glycosylation of surface proteins that are involved in attachment. Strain R301 lacked the plasmid genes and induces disease in rheas but not in pigs [9], hence suggesting that there is species-specificity in the colonisation process.

There was no evidence that other genes on the plasmid were correlated with virulence. Some other chromosomal genes of unknown function were absent in some of the avirulent strains, but the lack of consistency did not suggest that they are important in the context of pathogenesis. It is possible that other genes not represented on the microarray may be associated with virulence, and might have had a different distribution in the two sets of isolates. A more detailed understanding could be obtained by undertaking whole genomic sequencing of the B. hyodysenteriae strains.

The products of the plasmid genes that were present in the virulent strains but absent in the other strains apparently influence the pathogenesis of SD, although gene expression was not investigated. Neither was it possible to specifically inactivate the identified genes to determine whether this affects the phenotype and behaviour of the strains when used to infect pigs, due to lack of easily applicable means to genetically manipulate $B$. hyodysenteriae in a targeted way. Further studies of this sort should be undertaken in the future to help understand the role of the gene products.

Of the predicted gene products, radical SAM dependent enzymes catalyze a diverse assortment of reactions involved in numerous important biological pathways in other bacteria, including the biosynthesis of a wide number of enzyme cofactors and secondary metabolites, anaerobic oxidations, biosynthesis and repair of DNA, and general bacterial metabolism. SAM also is important for the production of a universal quorum-sensing signal in bacteria that regulates pathogen-host interactions. Inhibition of this enzyme in other bacteria can lead to attenuation of virulence [15].

The $r f b$ genes shape the structure of the LOS O-antigens that are likely to be involved in host-defence mechanism. In most bacteria, the $r f b$ genes are found on the genome as a cluster and are believed to have been acquired through lateral gene transfer [16]. An $r f b A B C D$ gene cluster was 
present on the plasmid of all the $B$. hyodysenteriae strains tested in this study, however the additional $r f b C$ gene on the plasmid of the virulent strains might contribute to virulence. Both $r f b C$ genes possess the dTDP-4-dehydrorhamnose 3,5-epimerase conserved domain, but are only $52 \%$ similar at the protein level. B. hyodysenteriae LOS is considered to contribute to virulence as it has various biological effects in vitro and in vivo [17-21].

Glycosyl transferases play an important role in pathogenicity of most pathogenic bacteria [22-24]. They catalyse the transfer of sugar moieties to acceptor substrates such as oligosaccharides, monosaccharides, proteins and lipids. Protein glycosylation can have roles in host interactions, protection against proteolytic cleavage, protein assembly, antigenic variation, and protective immunity. Glycosyl transferases are highly specific with respect to both the donor sugar and acceptor, and to date the exact specificities of most have not been determined [25].

Overall the findings of this study indicate that a block of specific genes on the B. hyodysenteriae plasmid are involved in facilitating colonisation and development of disease. Isolates lacking these genes can be predicted to have a lower pathogenic potential in pigs than those that have the genes.

\section{Competing interests}

The authors have long standing collaborations with animal health companies in the development of vaccines and novel diagnostic tests for swine dysentery.

\section{Authors' contributions}

TL participated in conceiving the study, participated in its design and coordination, helped perform the experiments, helped interpret the results and helped to draft the manuscript. NP participated in the design of the study, helped perform the experiments and helped with interpreting the results. JT provided clinical isolates and helped to interpret the results. DH participated in conceiving the study, participated in its design and coordination, helped interpret the results and helped to draft the manuscript. All authors read and approved the final manuscript.

\section{Acknowledgements}

The authors thank Novartis Animal Health for financial support for this project. Professor Matthew Bellgard kindly read and commented on the article.

\section{Author details}

'School of Veterinary and Life Sciences, Murdoch University, Murdoch, Western Australia 6150, Australia. ${ }^{2}$ Scottish Agricultural College Veterinary Services, Bush Estate, Penicuik, Midlothian, Scotland, UK

Received: 30 October 2014 Accepted: 4 December 2014

Published online: 16 December 2014

\section{References}

1. Hampson DJ: Brachyspiral colitis. In Diseases of Swine. 10th edition. Edited by Zimmerman JJ, Karriker LA, Ramirez A, Schwartz KJ, Stevenson GW. Ames, lowa, USA: Wiley-Blackwell Publishing; 2012:680-696.

2. Pluske JR, Siba PM, Pethick DW, Durmic Z, Mullan BP, Hampson DJ: The incidence of swine dysentery in pigs can be reduced by feeding diets that limit the amount of fermentable substrate entering the large intestine. J Nutr 1996, 126:2920-2933.

3. Siba PM, Pethick DW, Hampson DJ: Pigs experimentally infected with Serpulina hyodysenteriae can be protected from developing swine dysentery by feeding them a highly digestible diet. Epidemiol Infect 1996, 116:207-216.
4. Thomsen LE, Knudsen KE, Jensen TK, Christensen AS, Møller K, Roepstorff A: The effect of fermentable carbohydrates on experimental swine dysentery and whip worm infections in pigs. Vet Microbio/ 2007, 119:152-163.

5. Lysons RJ, Lemcke RM, Bew J, Burrows MR, Alexander TJL: An avirulent strain of Treponema hyodysenteriae isolated from herds free of swine dysentery. In Proceedings of the $7^{\text {th }}$ International Pig Veterinary Society Congress: 26-31 July 1982. Mexico City, Mexico: The Society; 1982:40.

6. Jensen NS, Stanton TB: Comparison of Serpulina hyodysenteriae B78, the type strain of the species, with other $S$. hyodysenteriae strains using enteropathogenicity studies and restriction fragment length polymorphism analysis. Vet Microbiol 1993, 36:221-231.

7. Achacha M, Messier S, Mittal KR: Development of an experimental model allowing discrimination between virulent and avirulent isolates of Serpulina (Treponema) hyodysenteriae. Can J Vet Res 1996, 60:45-49.

8. Thomson JR, Murray BP, Henderson LE, Moore L, Meikle CS: Virulence studies in porcine Brachyspira species by experimental challenge of pigs. Eddleston, Scotland: Proceedings of the Second International Conference on Colonic Spirochaetal Infections in Animals and Humans; 2003.

9. Stanton TB, Jensen NS, Bosworth BT, Kunkle RA: Evaluation of the virulence of rhea S. hyodysenteriae strains for swine. First International Virtual Conference on Infectious Diseases of Animals: Ames, lowa, USA. 1997.

10. Bellgard MI, Wanchanthuek P, La T, Ryan K, Moolhuijzen P, Albertyn Z, Shaban B, Motro Y, Dunn DS, Schibeci D, Hunter A, Barrero R, Phillips ND, Hampson DJ: Genome sequence of the pathogenic intestinal spirochete Brachyspira hyodysenteriae reveals adaptations to its lifestyle in the porcine large intestine. PLoS One 2009, 4:e4641.

11. La T, Phillips ND, Wanchanthuek P, Bellgard MI, O'Hara AJ, Hampson DJ: Evidence that the $36 \mathrm{~kb}$ plasmid of Brachyspira hyodysenteriae contributes to virulence. Vet Microbio/ 2011, 153:150-155.

12. Kunkle RA, Harris DL, Kinyon JM: Autoclaved liquid medium for propagation of Treponema hyodysenteriae. J Clin Microbiol 1986, 24:669-671.

13. La T, Phillips ND, Hampson DJ: Development of a duplex PCR assay for detection of Brachyspira hyodysenteriae and Brachyspira pilosicoli in pig feces. J Clin Microbiol 2003, 41:3372-3375.

14. Gene Expression Omnibus Public Repository [http://www.ncbi.nlm.nih. gov/geo/]

15. Parveen N, Cornell KA: Methylthioadenosine/S-adenosylhomocysteine nucleosidase, a critical enzyme for bacterial metabolism. Mol Microbiol 2011, 79:7-20.

16. Reeves PP, Wang L: Genomic organization of LPS-specific loci. Curr Top Microbiol Immunol 2002, 264:109-135

17. Nuessen ME, Birmingham JR, Joens LA: Biological activity of a lipopolysaccharide extracted from Treponema hyodysenteriae. Infect Immun 1982, 37:138-142.

18. Greer JM, Wannemuehler MJ: Pathogenesis of Treponema hyodysenteriae: induction of interleukin-1 and tumour necrosis factor by a treponemal butanol/water extract (endotoxin). Microb Pathog 1989, 7:279-288.

19. Nibbelink SK, Sacco RE, Wannemuehler MJ: Pathogenicity of Serpulina hyodysenteriae: in vivo induction of tumor necrosis factor and interleukin-6 by a serpulinal butanol/water extract (endotoxin). Microb Pathog 1997, 23:181-187.

20. Nuessen ME, Joens LA, Glock RD: Involvement of lipopolysaccharide in the pathogenicity of Treponema hyodysenteriae. J Immunol 1983, 131:997-999.

21. Nibbelink SK, Wannemuehler MJ: Susceptibility of inbred mouse strains to infection with Serpula (Treponema) hyodysenteriae. Infect Immun 1991, 59:3111-3118

22. Power PM, Roddam LF, Rutter K, Fitzpatrick SZ, Srikhanta YN, Jennings MP. Genetic characterization of pilin glycosylation and phase variation in Neisseria meningitidis. Mol Microbiol 2003, 49:833-847.

23. Szymanski CM, Wren BW: Protein glycosylation in bacterial mucosal pathogens. Nat Rev Microbiol 2005, 3:225-237.

24. Zhou M, Wu H: Glycosylation and biogenesis of a family of serine-rich bacterial adhesins. Microbiology 2009, 155:317-327.

25. Lundborg M, Modhukur V, Widmalm G: Glycosyltransferase functions of $E$. coli O-antigens. Glycobiology 2010, 20:366-368.

doi:10.1186/s13567-014-0131-6

Cite this article as: La et al:: Absence of a set of plasmid-encoded genes is predictive of reduced pathogenic potential in Brachyspira hyodysenteriae. Veterinary Research 2014 45:131. 